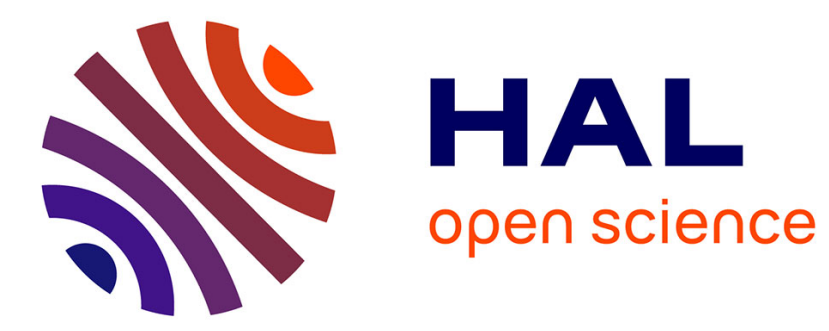

\title{
An intermittently served discrete time queue with applications to meteor scatter communications
}

\author{
P.J.B. King, I. Mitrani, Philippe Robert
}

\section{To cite this version:}

P.J.B. King, I. Mitrani, Philippe Robert. An intermittently served discrete time queue with applications to meteor scatter communications. RR-0712, INRIA. 1987. inria-00075840

\section{HAL Id: inria-00075840 \\ https://hal.inria.fr/inria-00075840}

Submitted on 24 May 2006

HAL is a multi-disciplinary open access archive for the deposit and dissemination of scientific research documents, whether they are published or not. The documents may come from teaching and research institutions in France or abroad, or from public or private research centers.
L'archive ouverte pluridisciplinaire HAL, est destinée au dépôt et à la diffusion de documents scientifiques de niveau recherche, publiés ou non, émanant des établissements d'enseignement et de recherche français ou étrangers, des laboratoires publics ou privés. 


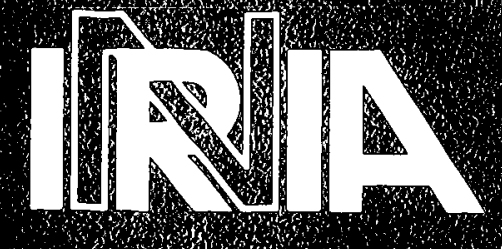

UNI E DE: RECWEROM NRHA MOOOH WNOURT

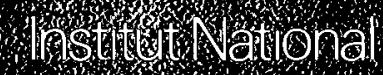
(r. 1 enerene 1: 4. et en arutomatíque

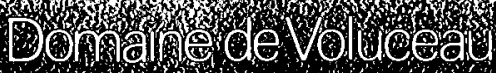

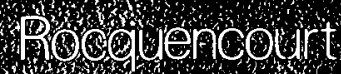

\section{.}

\section{Rapports de Recherche}

$\mathrm{N}^{\circ} \mathbf{7 1 2}$

\section{AN INTERMITTENTLY SERVED DISCRETE TIME QUEUE WITH APPLICATIONS TO METEOR SCATTER COMMUNICATIONS}




\title{
An Intermittently Served Discrete Time Queue with
}

\author{
Applications to Meteor Scatter Communications
}

\section{Etude d'une file d'attente a temps discret avec interruptions}

\author{
Peter J.B. King \\ Heriot-Watt, University Edinburgh, EH1 2JH, Scotland \\ Isi Mitrani ${ }^{1}$ \\ ATET Bell Laboratories, Murray Hill, NJ 07974 \\ Philippe Robert ${ }^{2}$ \\ INRIA, 78159 Le Chesnay, France
}

\begin{abstract}
A discrete time single server queue with service interruptions is analyzed in the steady-state under general assumptions. The main motivation for the study is the performance evaluation of a communication protocol using ionized layers created by meteors. The analysis yields the joint distribution of the queue size and the remaining duration of the current operative or inoperative period. The solution takes a particularly simple form in the case where the operative periods have a rational generating function.
\end{abstract}

\section{Résumé}

Nous étudions une file d'attente a temps discret avec interruptions sous des hypothèses générales. La principale motivation de cette étude est l' évaluation d'un protocole de communication simple utilisant les couches ionisées de l'atmosphère créees par les météores. Les interruptions de transmission interviennent lorsque la densité de matière ionisée est en dessous d'une valeur critique. Sous des hypothèses probabilistes nous obtenons la loi jointe du nombre de messages en attente de transmission et de la durée résiduelle de la période d'activité ou de non activité. La solution a une expression particulièrement simple dans le cas ou les périodes d'activité ont une fonction génératrice rationnelle.

1 On leave of absence from the University of Newcatle upon Tyne, NE1 7RU, England

2 On short term visits to the University of Newcastle upon Tyne and AT\&T Bell Laboratories.

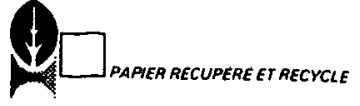




\section{Introduction}

A rather unusual approach to packet radio communications has started attracting attention in recent years. It is based on a phenomenon called 'meteor scatter'. Meteors passing through the upper reaches of the atmosphere (at heights of $100-150 \mathrm{~km}$ ) create ionized layers which act as reflecting mirrors for VHF signals. Two stations at a distance greater than that of the direct VHF range can use these layers to communicate with each other, as illustrated in figure 1.

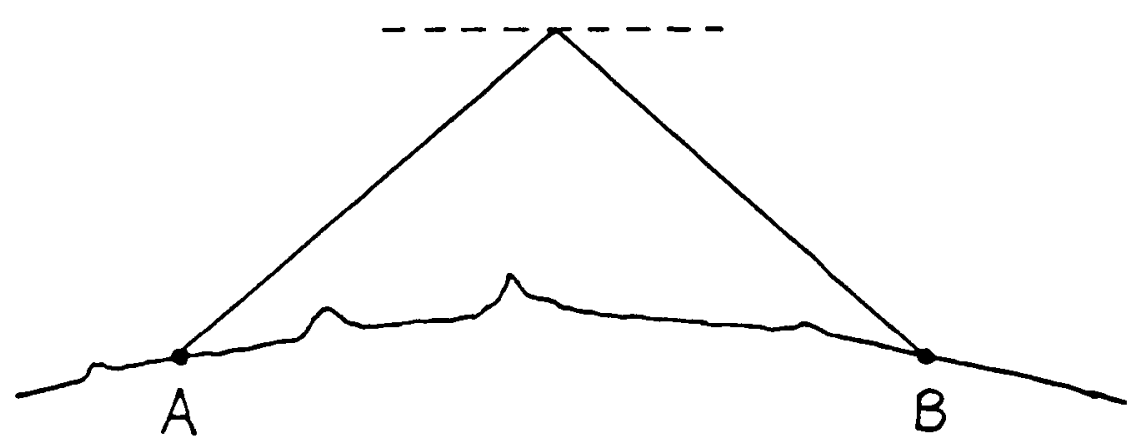

Figure 1.

We shall refer to the periods during which a suitable reflecting layer is available, or unavailable, as 'operative' and 'inoperative' periods, respectively. This emphasizes the analogy which exists between a medium such as meteor scatter, and a server that breaks down from time to time. In the 'meteor server' case, the operative periods last for about 0.5 seconds, and the inoperative ones for about 10 seconds. For a more detailed discussion of this phenomenon see, for example, $[2,8]$. 
Consider two stations which transmit at different frequencies, so that a message from $A$ to $B$, and one from $B$ to $A$, can be sent at the same time without interference. During inoperative periods, both stations continue to send a probing signal to each other. As soon as $A$ receives $B$ 's probing signal, it knows that an operative period is under way, and can start sending packets of information. Each of those packets is acknowledged by $B$. When the acknowledgements stop arriving, station $A$ realizes that the operative period has terminated and stops sending packets. However, a certain number of packets that were en route between $A$ and the ionized layer when the latter disappeared, are lost and have to be resent during the next operative period. Along with the probing signal that $A$ receives at the beginning of a new operative period is also an indication of the last packet successfully received by $B$.

The situation is completely symmetrical with respect to station $B$.

Our aim is to study the steady-state behavior of this protocol and evaluate its performance. To that end, we shall construct and analyze a discrete-time model of the system. Models of similar type (apart from the assumption concerning the wasted portion of an operative period) have been studied by Bruneel $[3,4]$. Our approach involves the analysis of a two-dimensional Markov chain. We obtain, under very general assumptions, the steady-state joint distribution of the number of packets awaiting transmission at a station and the remaining duration of the current operative or inoperative period. The numerical implementation of the solution is discussed. 
It should be pointed out that the applicability of the model is by no means restricted to the meteor scatter problem. Discrete-time queues with server interruptions occur often in computing and communications.

\section{The model}

The time axis is divided into slots of length 1, a slot being the time required to send a single packet. From the viewpoint of one of the stations, say station $A$, those slots belong to alternating operative and inoperative periods (an operative period starts with the reception of a probing signal from $B$, and ends when $B$ 's acknowledgements cease; because of propagation delays, that period does not coincide with the actual presence of an ionized layer). Packets sent during an operative period are received successfully and correctly, except if they are sent during the last $M$ slots of the period; in that case, they are lost. The time slots are illustrated in figure 2.

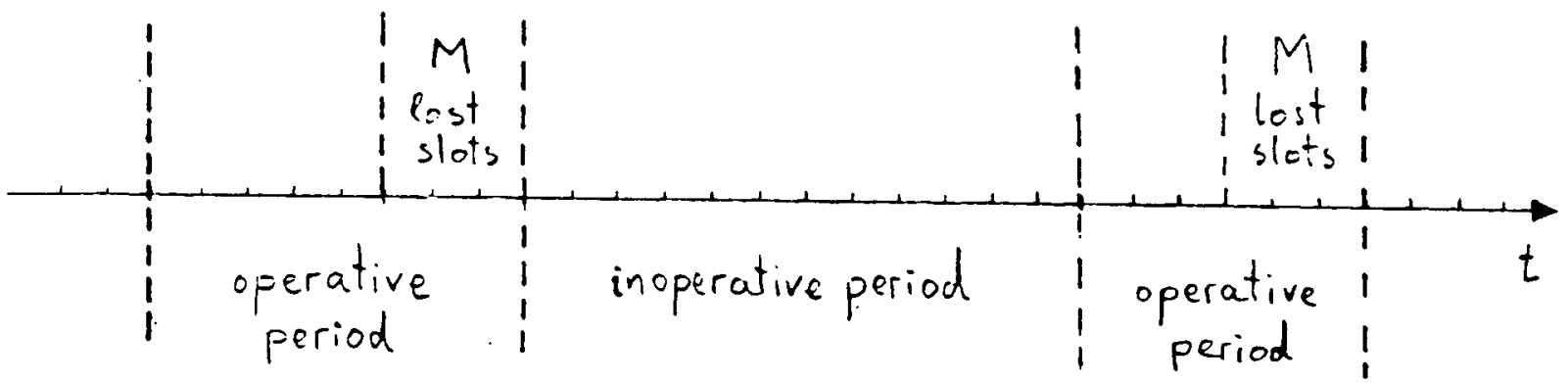

Figure 2. 
Let $\xi_{n}$ be the number of packets submitted for transmission at station $A$ during slot $n$ ( $\xi_{n}$ can also be thought of as the length of the message submitted in slot $n$ ). That random variable is independent of $n$, of the system state, and of past history. It has some arbitrary distribution, with generating function $a(z)$ :

$$
a(z)=\sum_{i=0}^{\infty} a_{i} z^{i}
$$

where $a_{i}=P\left(\xi_{n}=i\right) ; i=0,1, \ldots$. There is no bound on the number of packets that may be queued, awaiting transmission. The queue is served in FIFO order.

The length of an operative period (measured in number of slots) is denoted by $\eta$. Consecutive operative periods have independent and identically distributed lengths, with generating function $b(z)$ :

$$
b(z)=\sum_{i=1}^{\infty} b_{i} z^{i} .
$$

where $b_{i}=P(\eta=i) ; i=1,2, \ldots$. The length of an inoperative period is denoted by $\zeta$. Initially, these periods will be assumed geometrically distributed, with mean $1 / q$ slots. Thus, every slot of an inoperative period is followed by a new operative period with probability $q$. This amounts to assuming that the meteors arrive in a Poisson stream, which is not unreasonable. The generating function corresponding to that distribution, $w(z)$, is equal to

$$
w(z)=\frac{q z}{1-(1-q) z}
$$

However, the geometric assumption is not essential for the analysis. This is demonstrated in section 5 , where $\zeta$ may have an arbitrary distribution. 
The state of the system at the beginning of the n'th slot is described by a pair of integers, $\left(X_{n}, Y_{n}\right)$, where $X_{n}$ is the number of packets queued for transmission at station $A$ and $Y_{n}$ is the number of slots remaining in the current operative period, or 0 if the current period is inoperative. The above assumptions ensure that $\left(X_{n}, Y_{n}\right)$ is a Markov chain. In one slot, the packet queue size increases by the number of new arrivals, and decreases by 1 if a packet is transmitted successfully. The remaining portion of an operative period decreases by 1 throughout the life of that period, stays at 0 during the ensuing inoperative period and jumps to the size of the next operative period when the latter starts. Thus, the evolution of the system state is described by the following two relations:

$$
\begin{aligned}
& X_{n+1}=X_{n}+\xi_{n}-I\left(X_{n}>0\right) I\left(Y_{n}>M\right) \\
& Y_{n+1}=\left(Y_{n}-1\right) I\left(Y_{n}>0\right)+\eta I\left(Y_{n}=0\right) I(S),
\end{aligned}
$$

where $I(e)$ is the indicator function of the event $e: I(e)=1$ if $e$ occurs and $I(e)=0$ if it does not. The event $S$ occurs when a new operative period starts.

We are interested in the joint steady-state distribution, $p_{i j}$, of the packet queue size and the residual operative period:

$$
p_{i j}=\lim _{n \rightarrow x} P\left(X_{n}=i, Y_{n}=j\right)
$$

To establish the condition for existence of equilibrium, note that the average length of an 'operative-inoperative' cycle is $E(\eta)+\frac{1}{q}$, and that, in each such cycle, the average number of slots during which packets can be transmitted is $E\left[(\eta-M)^{+}\right]$, where $(\eta-M)^{+}=\max (\eta-M, 0)$. Hence, the maximum throughput that can be achieved is equal to $E\left[(\eta-M)^{+}\right] /\left[E(\eta)+\frac{1}{q}\right]$ packets 
per slot. Consequently, it is easily seen that the system is stable if, and only if, the average number of arrivals per slot is less than that quantity:

$$
E(\xi)<\frac{E\left[(\eta-M)^{+}\right]}{E(\eta)+\frac{1}{q}} .
$$

This condition will be assumed to bold.

Relations (4) imply that the probabilities $p_{i j}$ satisfy the following set of balance equations:

$$
\begin{aligned}
& p_{i j}=\sum_{k=0}^{i} a_{k}\left[p_{i+1-k, j+1}+q b_{j} p_{i-k, 0}\right]+a_{i} p_{0, j+1} ; i \geq 0, j \geq M, \\
& p_{i j}=\sum_{k=0}^{i} a_{k}\left[p_{i-k, j+1}+q b_{j} p_{i-k, 0}\right] ; i \geq 0,0<j<M, \\
& p_{i 0}=\sum_{k=0}^{i} a_{k}\left[p_{i-k, 1}+(1-q) p_{i-k, 0}\right] ; i \geq 0 .
\end{aligned}
$$

Introducing the generating functions

$$
g_{j}(x)=\sum_{i=0}^{\infty} p_{i j} x^{i}
$$

equations (7) can be written as

$$
\begin{aligned}
& g_{j}(x)=a(x)\left[\frac{g_{j+1}(x)-p_{0, j+1}}{x}+q b_{j} g_{0}(x)+p_{0, j+1}\right] ; j \geq M \\
& g_{j}(x)=a(x)\left[g_{j+1}(x)+q b_{j} g_{0}(x)\right] ; 0<j<M \\
& g_{0}(x)=a(x)\left[g_{1}(x)+(1-q) g_{0}(x)\right]
\end{aligned}
$$

These relntinns in turn, can be replaced by a single equation involving the bivariate ourating function 


$$
G(x, y)=\sum_{i=0}^{\infty} \sum_{j=0}^{\infty} p_{i j} x^{i} y^{j}=\sum_{j=0}^{\infty} g_{j}(x) y^{j}
$$

Multiplying (8) by $y^{j}$ and summing, we get, after some algebra,

$$
\begin{aligned}
& {[x y-a(x)] G(x, y)} \\
& =a(x)\left\{(x-1)\left[\sum_{j=1}^{M} g_{j}(x) y^{j}+y^{M} h(y)\right]-g_{0}(x)+x y g_{0}(x)[1-q+q b(y)]\right\} .
\end{aligned}
$$

where $h(y)$ is the generating function of the remaining number of useful slots in an operative period, when the packet queue is empty:

$$
h(y)=\sum_{j=M+1}^{\infty} p_{0 j} y^{j-M}
$$

In the right-hand side of (9) there are, apparently, $M+2$ unknown functions: $h(y)$ and $g_{j}(x)$, for $j=0,1, \ldots, M$. However, $M$ of the latter can be expressed in terms of $g_{0}(x)$ by using (8), for $0<j<M$. Then $G(x, y)$ would be given in terms of just two unknown fünctions of one variable each, $g_{0}(x)$ and $h(y)$.

The following observation will allow us to determine the unknowns: if $x$ and $y$ are such that

(i) $|x| \leq 1$ and $|y| \leq 1$ (so that $G(x, y)$ is finite), and

(ii) $x y-a(x)=0$,

then the right-hand side of (9) must vanish. This yields an equation which, after eliminating $g_{j}(x)$, for $j=1,2, \ldots, M$, can be written as 


$$
\begin{aligned}
& \frac{(1-x)}{q} w(a(x)) a(x)^{M-1} h(y)= \\
& \left\{w(a(x))\left[\sum_{j=1}^{M-1} a(x)^{j} b_{j}+a(x)^{M} b^{*}(y)\right]-1\right\} g_{0}(x) ;|x| \leq 1,|y| \leq 1, x y=a(x),
\end{aligned}
$$

where $w(z)$ is given by (3) and $b^{*}(z)$ is the generating function of $(\eta-M)^{+}$:

$$
b^{*}(z)=\sum_{j=M}^{\infty} b_{j} z^{j-M}
$$

In the next section it will be shown that there are indeed points for which (10) holds. Moreover, that equation will supply enough information to enable us to determine both $h(y)$ and $g_{0}(x)$.

\section{Analysis of the functional equation (10)}

We begin by examining the region of validity of (10), i.e., the set of points such that $|x| \leq 1,|y| \leq 1$ and $x y=a(x)$. Note first that if $x$ is on the unit circle, $|x|=1$, then $y=\frac{a(x)}{x}$ is in the unit disk. This is because $|y|=\left|\frac{a(x)}{x}\right|=|a(x)| \leq 1$. Thus, the mapping $y(x)=\frac{a(x)}{x}$ takes the unit circle of the $x$-plane onto some closed contour, $C_{y}$, which lies entirely in the unit disk of the $y$-plane. That contour touches the unit circle at the point $y(1)=1$, and, in general, only there (figure $3 a$ ).

On the other hand, if $y$ is on, or outside the unit circle, $|y| \geq 1$, then there is exactly one $x$ in the unit disk such that $x y=a(x)$. This is seen by applying Roucbe's theorem to the difference $x y-a(x)$. When $|x|=1$ we have $|a(x)| \leq|x y|$, with equality only for $x=1$ and $y=1$. Since $x-a(x)$ increases 
at $x=1$ (because $a^{\prime}(1)<1$ ), the circle can be deformed slightly around the point 1 , so that the inequality becomes strict everywhere. Hence, $x y-a(x)$ has the same number of roots in the unit disk as $x y$, i.e. exactly one root. Denote that root by $x(y)$. The exterior of the unit disk of the $y$-plane is thus mapped by $x(y)$ onto the interior of some closed contour, $C_{x}$, which lies entirely in the unit disk of the $x$-plane and touches the corresponding unit circle at the single point $x(1)=1$ (figure $3 b$ ).

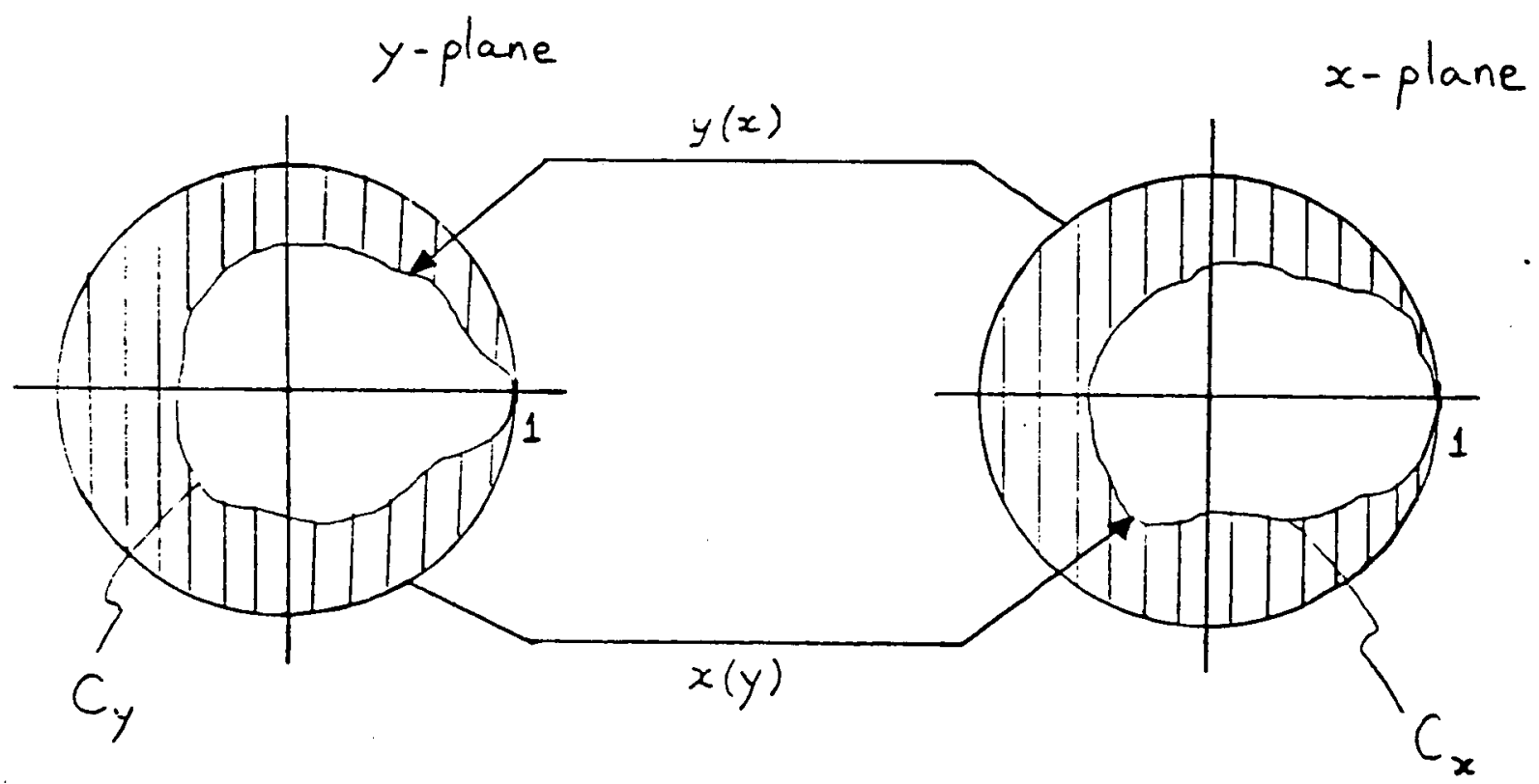

(a)

(b)

Figure 3.

The above arguments imply that when $x$ is between the unit circle and $C_{x}$ (the shaded region in figure $3 \mathrm{~b}$ ), $y(x)=\frac{a(x)}{x}$ is between the unit circle and $C_{y}$ (the shaded region in figure $3 \mathrm{a})$. For such pairs $(x, y(x))$, equation (10) holds. 
Now, let us rewrite (10) in the form

$$
h(y)=\phi(x) \frac{e(x)-1}{1-x} ; \quad x y=a(x)
$$

where

$$
\phi(x)=\frac{q g_{0}(x)}{w(a(x)) a(x)^{M-1}}
$$

and

$$
e(x)=w(a(x))\left[\sum_{j=1}^{M-1} a(x)^{j} b_{j}+a(x)^{M} b^{*}\left(\frac{a(x)}{x}\right)\right]
$$

Note that $\phi(x)$ is analytic in the entire unit disk. This is because, according to (10), every zero of $w(a(x)) a(x)^{M-1}$ is a zero of $g_{0}(x)$ with the same multiplicity.

To determine the unknown functions, we start by considering equation (11) for $y$ on the unit circle, $|y|=1$, and $x=x(y)$ on the curve $C_{x}$. The idea is to obtain a factorization of type Wiener-Hopf for the fraction $\gamma(y)=\frac{e(x(y))-1}{1-x(y)}$ (appearing in the right-hand side of (11)), on the unit circle. This takes the form

$$
\gamma(y)=\psi^{+}(y) / \psi^{-}(y) ;|y|=1
$$

where $\psi^{+}(y)$ is a function analytic in the interior of the unit disk, $|y|<1$, continuous on the circle $|y|=1$, and having no zeros for $|y| \leq 1$, except perhaps at points where $h(y)$ is zers; $\psi^{-}(y)$ is analytic in the exterior of the unit disk, $|y|>1$, continuous on the circle $|y|=1$, does not vanish for $|y| \geq 1$ and approaches a non-zero constant when $|y| \rightarrow \infty$.

General formulae for $\psi^{+}(y)$ and $\psi^{-}(y)$ are given in the appendix. A simple solution under a mildly restrictive assumption will be presented in the next section. 
Here we shall assume that the factorization (12) is given, and proceed to determine the functions $h(y)$ and $g_{0}(x)$.

Substituting (12) into (11) we get

$$
h(y)=\phi(x(y)) \frac{\psi^{+}(y)}{\psi^{-}(y)} ;|y|=1
$$

or

$$
\frac{h(y)}{\psi^{+}(y)}=\frac{\phi(x(y))}{\psi^{-}(y)} ;|y|=1
$$

From the definition of $\psi^{+}(y)$ and from the fact that $h(y)$ is a generating function, it follows that the left-hand side of (13) is analytic for $|y|<1$. Similarly from the definition of $\psi^{-}(y)$ and from the fact that $\phi(x)$ is analytic inside the unit disk $\left(x(y)\right.$ is inside $C_{x}$ when $\left.|y|>1\right)$, it follows that the righthand side of (13) is analytic for $|y|>1$ and is bounded by a constant at infinity. The equation itself, which is valid on the circle $|y|=1$, shows that the two sides are analytic continuations of each other. Therefore, together they define a function, $\chi(y)$, which is bounded and analytic in the whole plane:

$$
\chi(y)= \begin{cases}\frac{h(y)}{\psi^{+}(y)} & \text { if }|y| \leq 1 \\ \frac{\phi(x(y))}{\psi^{-}(y)} & \text { if }|y| \geq 1\end{cases}
$$

However, according to Liouville's theorem, $\chi(y)$ must be equal to some constant, $D$. Then the first part of (14) yields

$$
h(y)=D \psi^{+}(y) ;|y| \leq 1 .
$$


Similarly, the second part of (14) implies that

$$
\phi(x(y))=D \psi^{-}(y) ;|y| \geq 1
$$

or

$$
g_{0}(x)=\frac{D}{q} w(a(x)) a(x)^{M-1} \psi^{-}(y(x))
$$

where $x$ is in the interior of, or on $C_{x}$ (figure 3 ).

It is shown in the appendix that $\psi^{+}(y)$ and $\psi^{-}(y)$ contain a linear factor with unknown coefficients, $\left(c_{0}+c_{1} y\right)$. Thus, in order to specify the solution completely, two constants need to be determined, $D c_{0}$ and $D c_{1}$. The first of these is zero, since $h(0)=0$ (by the definition following equation (9)). The second is obtained from the normalizing condition, $G(1,1)=1$.

Note that both $h(y)$ and $g_{0}(x)$ can be continued outside the domains associated with (15) and (16), if desired. For instance (16), together with the functional equation (10), defines $h(y)$ for $|y|>1$. Similarly, (15) and (10) define $g_{0}(x)$ outside the contour $C_{x}$. For the purpose of calculating system performance measures, such continuations are not necessary.

It should be clear to anyone who reads the appendix, that the formulae given there do not lend themselves easily to numerical implementation. However, at the price of a very slight loss of generality, one can obtain a much more direct solution which is readily implementable. 


\section{Solution in the case when $b^{*}(y)$ is rational}

Assume that the generating function of $(\eta-M)^{+}$is a ratio of two arbitrary polynomials:

$$
b^{*}(y)=\frac{Q(y)}{R(y)} .
$$

This is not a serious restriction from the practical point of view.

Now the solution of the factorization problem (12) can be found rather simply. We look for $\psi^{+}(y)$ in the form of a ratio

$$
\psi^{+}(y)=\frac{P(y)}{R(y)}
$$

where $P(y)$ is some as yet unspecified polynomial. This form ensures that $\psi^{+}(y)$ is analytic for $|y|<1$, since $R(y)$, being the denominator of a generating function, cannot have any zeros in the unit disk. To meet the remaining requirements for $\psi^{+}(y)$, the polynomial $P(y)$ must also have no zeros in that region, except at $y=0$, where $h(y)$ vanishes.

Having chosen the form of $\psi^{+}(y)$, that of $\psi^{-}(y)$ is indicated by (12):

$$
\psi^{-}(y)=\frac{\psi^{+}(y)}{\gamma(y)}=\frac{P(y)[1-x(y)]}{R(y)[e(x(y))-1]} .
$$

We shall now determine $P(y)$ from the requirements that $\psi^{-}(y)$ is analytic outside the unit disk, does not vanish there, and tends to a constant when $|y| \rightarrow \infty$. Consider the denominator in (19):

$$
R(y)[e(x)-1]=w(a(x))\left[R(y) \sum_{j=1}^{M-1} a(x)^{j} b_{j}+a(x)^{M} Q(y)\right]-R(y),
$$


where $x=x(y)$ is in the interior of $C_{x}$ when $|y|>1$. Note first that when $|y| \rightarrow \infty$, the right-hand side of $(20)$ is on the order of $y^{d}$, where $d$ is the larger of the degrees of $Q(y)$ and $R(y)$. Therefore, in order that $\psi^{-}(y)$ has a finite and non-zero value at $y=\infty$, the polynomial $P(y)$ must be of degree $d$. Next, we have the following.

\section{Lemma.}

The function $R(y(x))[e(x)-1]$ has a single zero at $x=1$ and $d-1$ other zeros in the interior of $C_{x}$.

\section{Proof.}

The fact that $e(1)=1$ is easily verified by direct substitution. For $x \neq 1$, $x \in C_{x}$, it can be seen that $|e(x)|<1\left(|y(x)|=\left|\frac{a(x)}{x}\right|=1\right.$ on $\left.C_{x}\right)$. Also, the ergodicity condition (6) implies that $e^{\prime}(1)<0$. Therefore, if $C_{x}$ is deformed sufficiently slightly in the vicinity of $x=1$, by making it pass to the right of that point instead of through it, then the inequality $|e(x)|<1$ would hold on the entire contour.

One consequence of the above is that the variation of the argument of $e(x)-1$, as $x$ traverses the (modified) contour $C_{x}$, is 0 . Hence, $e(x)-1$ has as many zeros inside $C_{x}$ as it has poles. The same statement can be made for $R(y(x))=R\left(\frac{a(x)}{x}\right)$ (the latter has a pole of order $\operatorname{deg}(R)$ at $x=0$ ). On the other hand, a glance at (20), with $y=\frac{a(x)}{x}$, shows that $R(y(x))[e(x)-1]$ has a 
pole of order $d$ at $x=0$. Therefore, it has $d$ zeros inside $C_{x}$, including the one at $x=1$. All those points are, in fact, zeros of the function $e(x)-1$.

An immediate corollary of this lemma is that, considered as a function of $y$, the denominator in (19) has a zero at $y=1$ and $d-1$ other zeros, $y_{1}, y_{2}, \ldots, y_{d-1}$, which lie outside the unit disk. The zero at $y=1$ is neutralized by the factor $[1-x(y)]$ in the numerator, since $x(1)=1$. We are thus led to the conclusion that in order for $\psi^{-}(y)$ to be analytic for $|y|>1$, the polynomial $P(y)$ must vanish at each of the points $y_{i}(i=1,2, \ldots, d-1)$. Remembering that $P(0)=0$, we can write

$$
P(y)=c_{d} y\left(y-y_{1}\right)\left(y-y_{2}\right) \cdots\left(y-y_{d-1}\right),
$$

where $c_{d}$ is an unknown constant. But we already know that the solution for $\psi^{+}(y)$ and $\psi^{-}(y)$ that fits our problem is unique up to a constant. Therefore, $h(y)$ and $g_{0}(x)$ are obtained by substituting (21), (18) and (19) into (15) and (16), and determining the unknown constant $D c_{d}$ from the normalizing condition.

\section{General distribution of the inoperative periods}

The restriction that the inoperative periods of the server should be geometrically distributed can be removed quite easily. Suppose that the length of an inoperative period, $\zeta$, has some arbitrary distribution, $w_{i}=P(\zeta=i)$; $i=1,2, \ldots$, and generating function $w(z)$ :

$$
w(z)=\sum_{i=1}^{x} w_{i} z^{i}
$$

The system state can now be described by a triple, $(\delta, X, Y)$, where $\delta$ is 1 if the 
current slot is part of an operative period and 0 otherwise; $X$ is the number of packets present; $Y$ is the remaining number of slots in the current period (operative or inoperative). We can assume, without loss of generality, that $M=0$. The wasted portions of the operative periods can be included in the subsequent inoperative ones, since the latter can have a general distribution.

Denote the steady-state distribution of $(\delta, X, Y)$ by

$$
p_{k}(i, j)=P(\delta=k, X=i, Y=j) ; k=0,1, i, j=0,1, \ldots .
$$

These probabilities satisfy the following set of balance equations:

$$
\begin{gathered}
p_{1}(i, j)=\sum_{m=0}^{i} a_{m} p_{1}(i+1-m, j+1)+a_{i} p_{1}(0, j+1)+b_{j} \sum_{m=0}^{i} a_{m} p_{0}(i-m, 1) \\
p_{0}(i, j)=\sum_{m=0}^{i} a_{m} p_{0}(i-m, j+1)+w_{j}\left[\sum_{m=0}^{i} a_{m} p_{1}(i+1-m, 1)+a_{i} p_{1}(0,1)\right] \\
i, j=0,1, \ldots .
\end{gathered}
$$

Introducing the two generating functions

$$
g_{k}(x, y)=\sum_{i=0}^{\infty} \sum_{j=1}^{\infty} p_{k}(i, j) x^{i} y^{j-1} ; \quad k=0,1
$$

(22) can be transformed into two equations:

$$
\begin{aligned}
& {[x y-a(x)] g_{1}(x, y)=} \\
& a(x)\left\{x b(y) g_{0}(x, 0)+(x-1) g_{1}(0, y)-\left[g_{1}(x, 0)-(1-x) g_{1}(0,0)\right]\right\} . \\
& x[y-a(x)] g_{0}(x, y)=a(x)\left\{w(y)\left[g_{1}(x, 0)-(1-x) g_{1}(0,0)\right]-x g_{0}(x, 0)\right\} .
\end{aligned}
$$

Equation (25) can be used to express both $g_{0}(x, 0)$ and the term $\left\{g_{1}(x ; 0)-(i-x) g_{1}(0,0)\right]$ in terms of $g_{0}(x, 1)$. This is done by setting $y=a(x)$ and then $y=1$ in (25), and solving. Substituting the resulting 
expressions into (24) yields

$$
\begin{aligned}
{[x y-a(x)] g_{1}(x, y)=} & a(x)\left\{(x-1) g_{1}(0, y)\right. \\
& \left.-\frac{x[1-a(x)]}{a(x)[1-w(a(x))]}[1-w(a(x)) b(y)] g_{0}(x, 1)\right\} .
\end{aligned}
$$

This equation is very similar to (9). In particular, the kernel, [xy-a(x)], is the same. The two unknown functions are now $g_{1}(0, y)$, which plays the role of $h(y)$, and $g_{0}(x, 1)$, which plays the role of $g_{0}(x)$. In the region where $|x| \leq 1$, $|y| \leq 1$ and $x y=a(x)$, the right-hand side of (26) vanishes, which provides a relation between the unknown functions:

$$
y g_{1}(0, y)=\frac{[1-a(x)] g_{0}(x, 1)}{1-w(a(x))}\left[\frac{w(a(x)) b(y)-1}{1-x}\right] ; x y=a(x) .
$$

Equation (27) is of the same type as (11), with obvious analogues for $\phi(x)$ and $e(x)$. Its analysis proceeds as described in section 3. The special case when $b(y)$ is a rational function is treated as in section 4 .

\section{Numerical solution}

We shall outline here the implementation of the solution presented in section 4 . The first step is to find the $d-1$ roots, $x_{1}, x_{2}, \ldots, x_{d-1}$, of the equation $e(x)-1=0$ in the interior of the contour $C_{x}$. Note that the root $x=1$ is not included in that number. An efficient method for determining numerically the zeros of analytic and meromorphic functions is described in $[1,5,7]$.

The values $y_{i}$ appearing in (21) are obtained from 


$$
y_{i}=\frac{a\left(x_{i}\right)}{x_{i}} ; i=1,2, \ldots, d-1
$$

The remaining unknown constant, $D c_{d}$ is obtained from the condition $G(1,1)=1$. Setting $y=1$ in (9) and then letting $x \rightarrow 1$ gives, after an application of L'Hospital's rule,

$$
\sum_{j=0}^{M} g_{j}(1)+h(1)=1-a^{\prime}(1)=1-E(\xi) .
$$

The first term in the left-hand side of (29) is equal to the probability that either an inoperative period, or the last $M$-slot portion of an operative period is in progress. That probability is given by

$$
P\left(Y_{n} \leq M\right)=\frac{M+\frac{1}{q}}{E(\eta)+\frac{1}{q}}=\frac{1+q M}{1+q E(\eta)} .
$$

Combining (29) and (30) with (15), (18) and (21) yields an equation which determines the constant:

$$
D P(1)=R(1)\left[1-E(\xi)-\frac{1+q M}{1+q E(\eta)}\right]
$$

The two unknown functions, $h(y)$ and $g_{0}(x)$ are now completely determined by (15), (16), (18) and (19).

Suppose that the performance measure of interest is the average number of packets queued for transmission, $E(X)$. This is obtained by differentiating $G(x, 1)$ with respect to $x$ at $x=1$. Again one has to apply L'Hospital's rule (twice) in order to resolve the indeterminacy. The average response time for a packet, $W$, is riven by Little's result: $W=E(X) / E(\xi)$. 
The above solution procedure was implemented for several special cases of the model. The generating function of the arriving packets was taken as $a(x)=(1-\alpha)+\alpha x$. In other words, a single packet arrives in a slot with probability $\alpha$. The distribution of the operative periods is assumed to be an $m$-fold convolution of a geometric distribution:

$$
b(y)=\left[\frac{r y}{1-(1-r) y}\right]^{m}
$$

In figure 4 , the average packet queue size, $E(X)$, is plotted against the arrival rate, $\alpha$, for $m=1,2$ and 3 . The average length of the operative periods, $E(\eta)=\frac{m}{r}$, is held constant throughout. Also, the number of wasted slots, $M$, is always 1 .

The above assumptions imply that $d=m$. When $m=1$, the normalizing equation suffices to determine the single unknown constant. When $m=2$, there are two unknown constants and one (real) zero of $e(x)-1$ inside the unit circle. When $m=3$, two extra equations are provided by the two (complex conjugate) zeros of $e(x)-1$. More precisely, those two equations are provided by the real and the imaginary parts of one of the points.

Note that the effect of increasing $m$, while keeping $E(\eta)$ fixed, is to decrease the variance of the operative periods. As expected, this leads to lower average queue sizes. 


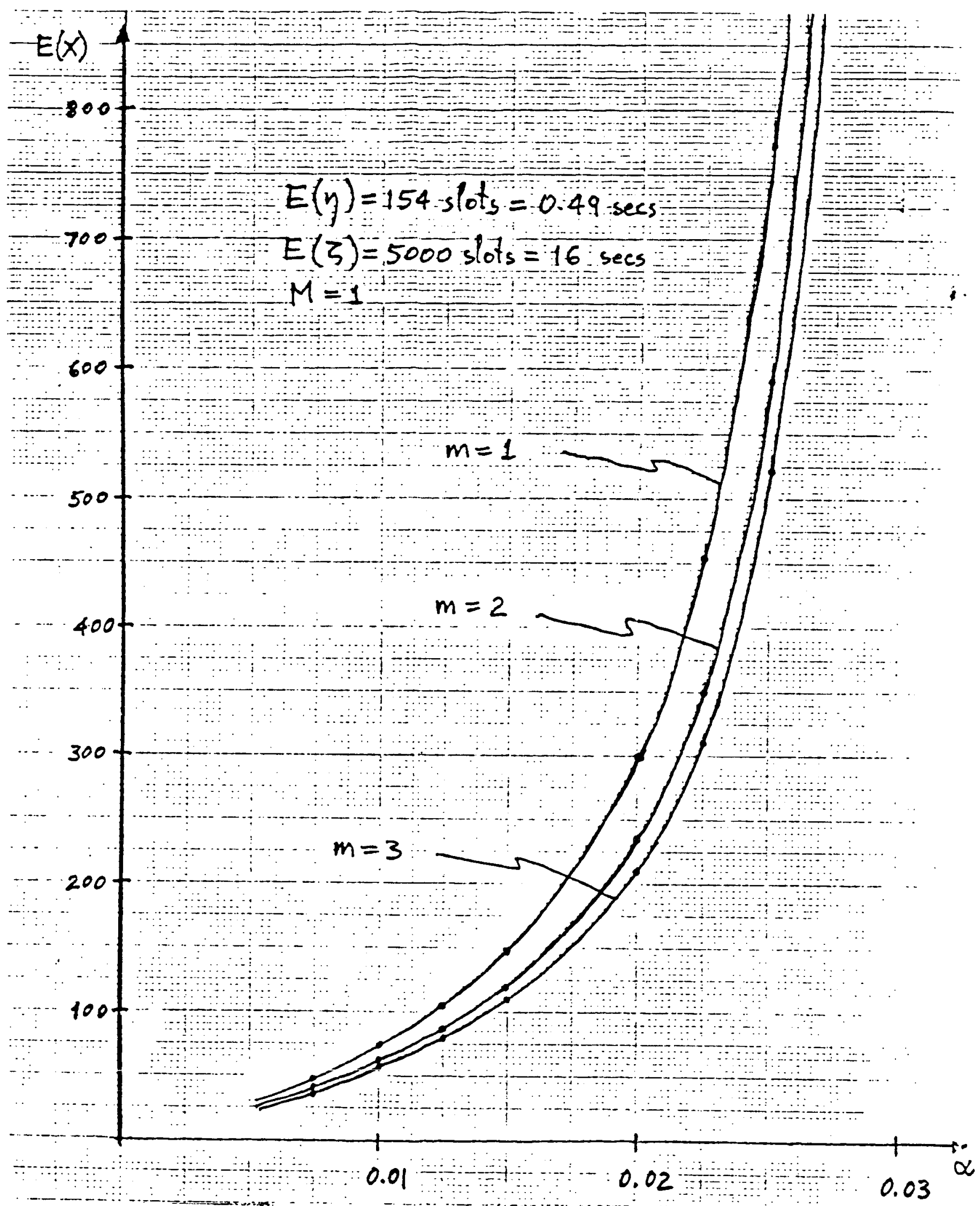

Figure 4. 


\section{REFERENCES}

[1] Abd-Ellal, L. F., Delves, L. M. and Reid, J. K., "A numerical method for locating the zeros and poles of a meromorphic function," in "Numerical methods for nonlinear algebraic equations" (ed. P. Rabinowitz) Gordon and Breach, 1970.

[2] Brown, D. W., "A physical meteor-burst propagation model and some significant results for communication system design," IEEE J. on Selected Areas in Comm., SAC-3, 5, pp. 745-755, 1985.

[3] H. Bruneel, "Analysis of an infinite buffer system with random server interruptions," Comput. \& Oper. Res., 11, 4, pp. 373-386, 1984.

[4] H. Bruneel, "A general treatment of discrete-time buffers with one randomly interrupted output line", European Journal of Oper. Res., 27, pp. $67-81,1986$.

[5] Delves, L. M. and Lyness, J. N., "A numerical method for locating the zeros of an analytic function," Mathematics of Computation, 21, 4, pp. 543-560, 1967.

[6] Gakhov, F. D., Boundary Value Problems, Pergamon Press, 1966.

[7] Lyness, J. N. and Delves, L. M., "On numerical contour integration round a closed contour," Mathematics of Computation, 21, 4, pp. 561-577, 1967.

[0] Oeting, J. D., "An analysis of meteor-burst communications for military 4pulations, IEEE Trans. Comm., COM-28, 9, pp. 1591-1601, 1980. 


\section{APPENDIX}

The problem of finding the two functions $\psi^{+}(y)$ and $\psi^{-}(y)$ is referred in the literature as the 'homogeneous Riemann boundary value problem' on the unit circle (see, for example, [6]). The existence and number of solutions depend on the 'index' of the function $\gamma(y)$. This last quantity is defined as the variation of the argument of $\gamma(y)$, as $y$ traverses the unit circle in anticlockwise direction, measured in units of $2 \pi$. Alternatively, it is equal to the number of zeros minus the number of poles of $\gamma(y)$ inside the unit circle.

To evaluate the index in our case, we shall deform the unit circle in the vicinity of point 1 , by making it pass slightly to the left of that point instead of through it. Then, as $y$ traverses the deformed circle, $x(y)$ describes a contour which coincides with $C_{x}$, except that it passes slightly to the right of point 1 instead of through it. Then, as we saw in the proof of the lemma in section 4 , the variation of the argument of $e(x(y))-1$ is 0 .

The index of $\gamma(y)$ on the deformed circle can be calculated as follows:

$$
\text { ind } \begin{aligned}
{[\gamma(y)] } & =\operatorname{ind}\left[\frac{e(x(y))-1}{1-x(y)}\right]=\operatorname{ind}[e(x(y))-1]-\operatorname{ind}[1-x(y)] \\
& =0-(-1)=1
\end{aligned}
$$

(the index of $1-x(y)$ is -1 because that function has no zeros and one pole inside the contour: $x(y)$ is outside $C_{x}$ when $|y|<1$ and $|x(y)|=\infty$ when $y=0$ ). Moreover, since a deformation of the contour which does not pass through any zeros or poles leaves the index unchanged, and since the point 1 is 
neither a zero nor a pole of $\gamma(y)$, we can assert that (A1) holds also on the unit circle.

When the index is 1 , the boundary problem has a solution which depends on two constants. It is given in terms of the piecewise analytic function $\Gamma(y)$, defined by the Cauchy integral

$$
\Gamma(y)=\frac{1}{2 \pi i} \int_{C_{1}} \frac{\ln [\gamma(s)]-\ln (s)}{s-y} d s,
$$

where $C_{1}$ is the unit circle. Let $\Gamma^{+}(y)$ be the part of $\Gamma(y)$ inside the unit circle and $\Gamma^{-}(y)$ be the part outside. Then the solution has the form (see [6])

$$
\psi^{+}(y)=\left(c_{0}+c_{1} y\right) e^{\Gamma^{+}(y)} ; \psi^{-}(y)=\left(\frac{c_{0}+c_{1} y}{y}\right) e^{\Gamma^{-}(y)}
$$

To find the limits of $\Gamma(y)$ as $y$ approaches a point on the unit circle from the inside or outside (e.g., in order to evaluate $\psi^{+}(1)$ ), one uses the formulae of Sokhotski-Plemelj:

$$
\begin{aligned}
& \Gamma^{+}(y)=\frac{1}{2} \beta(y)+\frac{1}{2 \pi i} \int_{C_{1}} \frac{\beta(s)}{s-y} d s ;|y|=1, \\
& \Gamma^{-}(y)=-\frac{1}{2} \beta(y)+\frac{1}{2 \pi i} \int_{C_{1}} \frac{\beta(s)}{s-y} d s ;|y|=1,
\end{aligned}
$$

where $\beta(y)=\ln (\gamma(y))-\ln (y)$; the singular integrals in (A4) and (A5) are taken in the sense of principal value. 
